\title{
OPEN Therapeutic potential of highly functional codon-optimized microutrophin for muscle-specific expression
}

Anna V. Starikova ${ }^{1,7}$, Victoria V. Skopenkova ${ }^{1,2,7}$, Anna V. Polikarpova ${ }^{1}$, Denis A. Reshetov ${ }^{3}$ Svetlana G. Vassilieva ${ }^{1}$, Oleg A. Velyaev ${ }^{1}$, Anna A. Shmidt ${ }^{1,2}$, Irina M. Savchenko ${ }^{1}$, Vladislav O. Soldatov ${ }^{4,5}$, Tatiana V. Egorova ${ }^{1,6 \bowtie}$ \& Maryana V. Bardina ${ }^{1,2,6}$

High expectations have been set on gene therapy with an AAV-delivered shortened version of dystrophin ( $\mu$ Dys) for Duchenne muscular dystrophy (DMD), with several drug candidates currently undergoing clinical trials. Safety concerns with this therapeutic approach include the immune response to introduced dystrophin antigens observed in some DMD patients. Recent reports highlighted microutrophin ( $\mu$ Utrn) as a less immunogenic functional dystrophin substitute for gene therapy. In the current study, we created a human codon-optimized $\mu U t r n$ which was subjected to side-by-side characterization with previously reported mouse and human $\mu$ Utrn sequences after rAAV9 intramuscular injections in $m d x$ mice. Long-term studies with systemic delivery of rAAV9$\mu U t r n$ demonstrated robust transgene expression in muscles, with localization to the sarcolemma, functional improvement of muscle performance, decreased creatine kinase levels, and lower immunogenicity as compared to $\mu \mathrm{Dys}$. An extensive toxicity study in wild-type rats did not reveal adverse changes associated with high-dose rAAV9 administration and human codon-optimized $\mu U t r n$ overexpression. Furthermore, we verified that muscle-specific promoters MHCK7 and SPc5-12 drive a sufficient level of rAAV9- $\mu$ Utrn expression to ameliorate the dystrophic phenotype in $\mathrm{mdx}$ mice. Our results provide ground for taking human codon-optimized $\mu$ Utrn combined with muscle-specific promoters into clinical development as safe and efficient gene therapy for DMD.

Duchenne muscular dystrophy (DMD) is a severe and progressive muscle-wasting disorder affecting one in 5000 boys $^{1}$. It is caused by mutations in the DMD gene leading to the loss of dystrophin, a large structural protein located beside the sarcolemma. Gene replacement therapy involving the systemic delivery of a shortened version of dystrophin (microdystrophin, $\mu \mathrm{Dys}$ ) through recombinant adeno-associated vectors (rAAV) represents a potential approach for DMD treatment ${ }^{2}$. Over 20 years of intensive research has allowed for the development of four rAAV- $\mu$ Dys vectors, which reached clinical trials (NCT03375164, NCT03362502, NCT03368742, and GNT0004). One of the encountered challenges was the generation of immune responses against epitopes in $\mu$ Dys, which may have contributed to a failure to detect dystrophin expression ${ }^{3}$. In light of $\mu$ Dys immunogenicity, utrophin has emerged as a promising target for innovative DMD therapy.

The utrophin protein is a functional paralog of dystrophin with $73 \%$ amino acid sequence identity ${ }^{4}$. Fulllength utrophin is highly expressed throughout the sarcolemma in fetal muscle. The level of utrophin at the muscle membrane declines during late embryonic stages and becomes restricted to the myotendinous and neuromuscular junctions as well as blood vessels in normal adult muscle ${ }^{5}$. Thereafter, its functions within the sarcolemma are performed by dystrophin, which connects the contractile apparatus and extracellular matrix through the dystrophin-associated glycoprotein complex (DAGC). Similar structure and overlapping localization

${ }^{1}$ Laboratory of Modeling and Gene Therapy of Hereditary Diseases, Institute of Gene Biology, Russian Academy of Sciences, Moscow, Russia 119334. ${ }^{2}$ Center for Precision Genome Editing and Genetic Technologies for Biomedicine, Institute of Gene Biology, Russian Academy of Sciences, Moscow, Russia 119334. ${ }^{3}$ Research Centre for Genetic Medicine, Moscow, Russia 117292. ${ }^{4}$ Department of Pharmacology and Clinical Pharmacology, Belgorod State National Research University, Belgorod, Russia 308007. ${ }^{5}$ Institute of Gene Biology, Core Facility Centre, Russian Academy of Sciences, Moscow, Russia 119334. ${ }^{6}$ Marlin Biotech LLC, Sochi, Russia 354340. ${ }^{7}$ These authors contributed equally: Anna V. Starikova and Victoria V. Skopenkova. ${ }^{\circledR}$ email: egorovatv@genebiology.ru 
A Utrophin

(400 kDa)

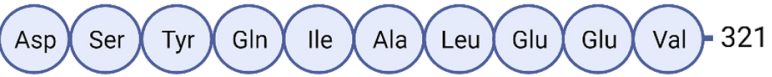

M- $\mu U$ trn

GAC TCC TAT CAA ATT GCT TTG GAG GAG GTT

$\mathrm{H}-\mu \mathrm{Utrn}$

GAC AGC TAT CAG ATT GCG TTG GAG GAA GTG

Figure 1. Design of $\mu$ Utrn-coding sequences. (A) Domain structure of full-length utrophin and $\mu U$ trn proteins. Domain configuration of $\mu$ Utrn $\Delta \mathrm{R} 4-\mathrm{R} 21 / \Delta \mathrm{CT}$ closely resembles microdystrophin R4-R23/ $\Delta \mathrm{CT}$ and consists of an N-terminal actin-binding domain, hinge 1 , spectrin-like repeats $1-3$, hinge 2 , spectrin-like repeat 22 , hinge 4 , and a cysteine-rich (CR) domain. (B) Alignment of the $\mu$ Utrn coding sequences used in this study. Fragments of spectrin-like repeat 1 coding sequences are shown for mouse $(M-\mu U t r n)$, human $(H-\mu U t r n)$, and codonoptimized human $(\mathrm{Hco}-\mu \mathrm{Utrn}) \mu \mathrm{Utrns}$. Codons optimized for expression in muscle are highlighted in bold red.

allow the use utrophin as a highly functional dystrophin substitute ${ }^{6,7}$. Thus, the upregulation of endogenous utrophin via small molecules ${ }^{8-10}$ artificial transcription factors ${ }^{11,12}$, and biglican therapy has been explored for DMD treatment ${ }^{13,14}$. rAAV-based therapies with a miniaturized form of utrophin represent another promising therapeutic approach for DMD.

The limited capacity of AAV vectors necessitates an effective expression cassette with a reduced length of the delivered gene. In 2003, Cerletti and colleagues ${ }^{15}$ performed adenovirus-mediated mini-utrophin gene transfer in Golden Retriever dogs with canine muscular dystrophy. Mini-Utrophin was a synthetic construct (AX107972), with $\mathrm{N}$ - and C-terminal regions derived from murine and human cDNA, respectively. Treatment reduced fibrosis and upregulated the expression of dystrophin-associated glycoproteins in affected muscle. In 2008, the Chamberlain lab designed a murine codon-optimized $\mu$ Utrn $\Delta \mathrm{R} 4-\mathrm{R} 21 / \Delta \mathrm{CT}$ (EU293093.1), which was identical to a previously reported truncated variant of dystrophin ${ }^{16,17}$. rAAV6- $\mu$ Utrn improved muscle histopathology and prolonged the lifespan of $m d x: u t r n^{-/-}$mice. In 2009, Sonnemann et al. ${ }^{18}$ showed that repeated systemic administration of $\Delta \mathrm{R} 4-21 \mu$ Utrn-TAT protein could attenuate the phenotype of $m d x$ mice. Kennedy et al. ${ }^{19}$ later confirmed that AAV9- $\mu$ Utrn $(\Delta \mathrm{R} 4-\mathrm{R} 21 / \Delta \mathrm{CT})^{17}$ could indeed alleviate skeletal and cardiac muscle pathology in D2/mdx mice. Recently, Song et al. ${ }^{20}$ carried out an extensive preclinical study with codon-optimized $\mu$ Utrn delivered to small and large animals via an rAAV9 vector, demonstrating the lower immunogenicity of $\mu$ Utrn compared to $\mu$ Dys. Banks et al. ${ }^{21}$ reported that rAAV6-CK8e- $\mu$ Utrn improved dystrophic pathophysiology, with fiber-type expression preferences.

Further, many studies of microdystrophin vectors have demonstrated that codon optimization can significantly increase transgene protein levels ${ }^{22,23}$. Muscle-specific promoters can provide a high, robust expression of the transgene in skeletal muscle, diaphragm, and heart, with limited activity in non-target tissues ${ }^{24}$. While such promoters are used in all clinical trials for microdystrophin deliver ${ }^{2}$, few studies have reported muscle-specific $\mu$ Utrn expression ${ }^{20,21}$.

In the present study, we sought to further explore the safety and efficacy of AAV-delivered $\mu$ Utrn constructs. Based on mouse $\mu$ Utrn configuration ${ }^{17}$, we designed a new human codon-optimized transgene and characterized it in the context of rAAV9-based administration in rodent models. $M d x$ mice were used to assess the efficacy of human codon-optimized $\mu$ Utrn in alleviating the dystrophic phenotype, and transgene immunogenicity was evaluated relative to that of a microdystrophin-coding construct. Extensive toxicity studies were performed in $m d x$ mice and Wistar rats following systemic delivery. Finally, we provide data on the expression and functional activity of codon-optimized $\mu$ Utrn driven by muscle-specific promoters.

\section{Results}

Intramuscular administration of rAAV9- $\mu$ Utrn leads to robust expression of $\mu$ Utrns and muscle function improvement in $\mathbf{m d x}$ mice. Previous studies provided compelling evidence that murine and human $\mu$ Utrns $\Delta$ R4-R21/ $\Delta$ CT (Fig. 1A) can functionally compensate for the lack of dystrophin in mouse models of DMD ${ }^{17,19-21}$. Building on their findings, we designed a human version of $\mu$ Utrn $\Delta \mathrm{R} 4-\mathrm{R} 21 / \Delta \mathrm{CT}$ and customized it through codon optimization in order to enhance expression in striated muscles and heart, as described in Materials and Methods (Fig. 1B). The human $\mu$ Utrn sequence had $37 \%$ optimal codons, and this index reached $100 \%$ after optimization (Supplementary Fig. S1). For direct comparison, the murine, human, and codon-optimized human $\mu U t r n$ cDNAs (designated in this study as M- $\mu U t r n, H-\mu U t r n$, and Hco- $\mu U t r n$, respectively) were cloned into the AAV vector expression cassette under a cytomegalovirus (CMV) promoter. As a control, a similar AAV construct with the cDNA of microdystrophin $\Delta \mathrm{R} 4-\mathrm{R} 23 / \Delta \mathrm{CT}^{16}$ was created. To produce 

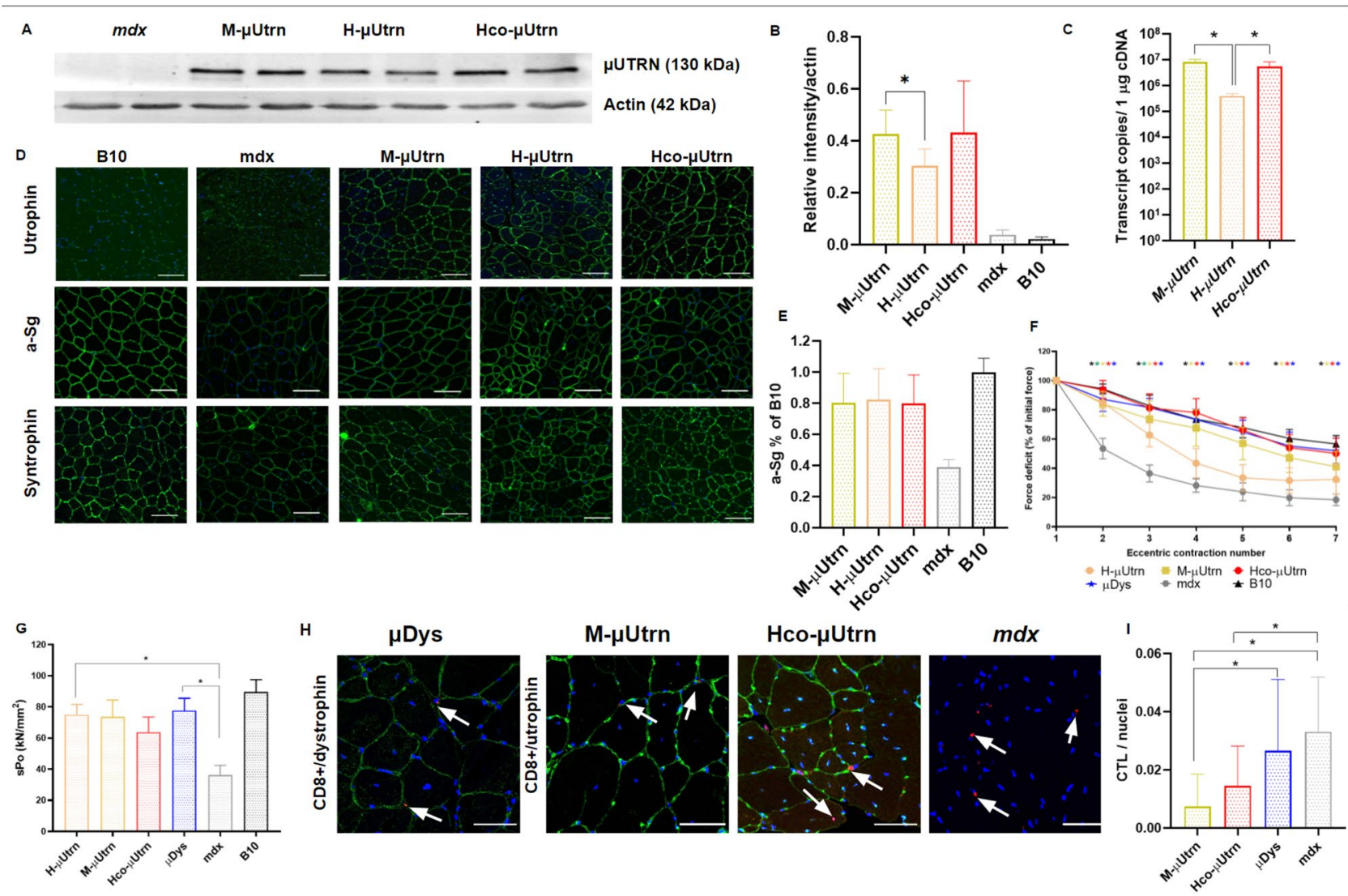

Figure 2. Intramuscular administration of $\mathrm{rAAV} 9-\mu \mathrm{Utrn}$ at dose $2 \times 10^{12} \mathrm{vg} / \mathrm{TA}$ muscle leads to robust transgene expression and improves the contractile function of the TA muscle in $m d x$ mice after 2 weeks post injection. (A) Western blot analysis of recombinant $\mu$ Utrn expression in tibialis anterior (TA) muscles, with $\alpha$-actin as the loading control. Unprocessed full-length blots are presented in Supplementary Figure S10. (B) Quantitation of $\mu$ Utrn expression determined via western blot. Data are presented as mean $\pm \mathrm{SD},{ }^{\star} P \leq 0.05$, $\mathrm{n}=4$ /group. (C) Analysis of $\mu$ Utrn transgene expression via RT-qPCR. (D) Representative images of native and recombinant utrophin as well as $\alpha$-sarcoglycan (a-Sg) and $\alpha 1$-syntrophin immunofluorescence in TA muscle cross-sections. Nuclei are counterstained with Hoechst 33,342 (blue). Scale bars, $100 \mu \mathrm{m}$. (E) Analysis of $\alpha$-sarcoglycan (a-Sg) expression levels in the sarcolemma of rAAV9- $\mu$ Utrn-treated muscle in comparison to that in untreated $m d x$ and B10 mice. Data are presented as the mean $\pm \mathrm{SD}, \mathrm{n}=4-10$ sections/group. (F) Percentage force drop following $20 \%$ eccentric contraction and (G) specific force of TA muscles from $m d x$ mice administered rAAV9- $\mu$ Utrn as compared to that in vehicle control mice. Values in $(\mathbf{C}, \mathbf{F})$, and $(\mathbf{G})$ are presented as the mean \pm SEM $(\mathrm{N}=8$ TA muscles for each group), and statistical significance was set at $P \leq 0.05$. (H) Representative images of CD8 + cytotoxic T-lymphocyte immunofluorescence (red dots marked with white arrows) in TA muscle sections. $\mu$ Utrn and $\mu$ Dys in the sarcolemma were stained in green, while nuclei were stained in blue. Scale bar, $50 \mu \mathrm{m}$. (I) Quantitation of CD8 + CTLs in TA muscle sections. CTL number normalized per nuclei, reflecting the cross-sectional area. Values are expressed as the mean $\pm S D, n=11-55$ sections analyzed per group.

rAAV viruses encoding $\mu$ Utrns (rAAV-CMV- $\mu$ Utrn), we used AAV serotype 9, which is currently being tested in clinical trials for microdystrophin delivery (NCT003362502, NCT03368742).

To characterize different $\mu U$ trn sequences in dystrophic mice, rAAV9-CMV- $\mu$ Utrn viruses were administered to 7 -week-old $m d x$ mice via intramuscular injections of $2 \times 10^{12} \mathrm{vg}$ into the tibialis anterior (TA) muscle. rAAV9$\mathrm{CMV}-\mu \mathrm{Dys}$ viruses were used as a control. The expression, potency, and immunogenicity of $\mu$ Utrns were assessed after 2 weeks. As expected, the CMV promoter drove robust mRNA expression in the muscle tissue for all three $\mu$ Utrn-coding vectors (Fig. 2 c). $H$ - $\mu U$ trn transcripts were the least abundant among $M-\mu U t r n$ and Hco- $\mu U t r n$. Recombinant utrophin production was confirmed via western blotting analysis with antibodies against the N-terminus of the utrophin protein (Fig. 2A). Quantification of the protein bands revealed lower expression levels for $\mathrm{H}-\mu \mathrm{Utrn}$ (Fig. 2B), in agreement with mRNA expression. Importantly, codon usage bias in Hco- $\mu$ Utrn did not compromise protein translation within murine muscles. Immunofluorescence analysis with antibodies against utrophin indicated that $\mu$ Utrn proteins successfully localized to the sarcolemma of muscle cells, where they can perform their function, substituting dystrophin protein in the DAGC of $m d x$ mice (Fig. 2D). Immunostaining of treated muscles with antibodies against DAGC proteins $\alpha$-sarcoglycan and al-syntrophin indicated that, in comparison with non-treated $m d x$ muscles, the levels of both increased considerably (Fig. 2D,E). 
Striking improvements of TA contractile properties were observed in rAAV9- $\mu$ Utrn-treated $m d x$ mice, with specific force increasing roughly twofold relative to that in untreated $m d x$ mice (Fig. $2 \mathrm{G}$ ). Similarly to $\mu \mathrm{Dys}$, $\mathrm{H}-\mu \mathrm{Utrn}$ exhibited a pronounced effect. All miniaturized proteins (H- $\mu \mathrm{Utrn}, \mathrm{M}-\mu \mathrm{Utrn}, \mathrm{Hco}-\mu \mathrm{Utrn}, \mu \mathrm{Dys})$ ensured muscle susceptibility to repeated eccentric contraction at levels comparable to those in control B10 mice (Fig. 2F). Force drop dynamics were indistinguishable between Hco- $\mu$ Utrn-treated, wild-type control, and $\mu$ Dys group muscles, indicative of the high functionality of the recombinant protein.

In order to verify the lower immunogenicity of utrophin, we assessed the presence of CD8 + cytotoxic T-lymphocytes (CTLs) in muscle sections. Lymphocytes were detected around muscle fibers in all analyzed groups (Fig. $2 \mathrm{H}$ ), which is typical for the dystrophic environment and has been previously investigated by other groups ${ }^{25}$. We observed a decrease in CTL count in M- $\mu$ Utrn- and Hco- $\mu$ Utrn-treated muscles compared to untreated $m d x$ muscles reflecting lowering of inflammation, while infiltration rate remained unchanged in the $\mu$ Dys-treated group (Fig. 2I). At the same time $\mu$ Dys-treated muscles showed similar functional recovery as $\mu$ Utrn-treated, indicating successful expression and functionality of all transgenes (Fig. $2 \mathrm{~F}, \mathrm{G}$ ). One can speculate that $\mu \mathrm{Dys}$ may be targeted by CTLs to a greater extent than $\mu$ Utrn, counterbalancing reduced dystrophy-associated infiltration as a consequence of treatment. Moreover, sections of Hco- $\mu$ Utrn-treated muscle exhibited more CTLs than observed for the M- $\mu$ Utrn group. A possible reason could be that the mice received human codon-optimized $\mu$ Utrn, which differs from endogenous murine utrophin. The cellular immune response may be triggered against the AAV capsid as well as the transgene product ${ }^{3,26}$. Since we used the rAAV9 vector for microgene delivery in all groups, we assume that the difference in the number of infiltrating T-cells was associated with the different transgenes. Found observations need further investigation at longer time points and in the context of systemic administration, as intramuscular AAV delivery is known to enhance immune responses ${ }^{27}$.

Side-by-side comparison of $\mu$ Utrn variants following intramuscular injections in $m d x$ mice revealed the potential of rAAV9-Hco- $\mu$ Utrn, which induced the robust expression of functional protein with lower immunogenicity. Taking into account the potential of microutrophins as a gene therapy for DMD, human codonoptimized $\mu$ Utrn was chosen for the subsequent experiments.

Systemic delivery of rAAV9-Hco- $\mu$ Utrn for long-term studies in $\mathbf{m d x}$ mice. In order to explore the systemic effects of transgene delivery, 6 -week-old $m d x$ mice were injected with rAAV9-Hco- $\mu$ Utrn at a dose $6 \times 10^{14} \mathrm{vg} \mathrm{kg}^{-1}$. The twenty-week study included functional testing throughout the experiment (the hanging wire test) and multiple terminal examinations such as transgene expression, muscle histopathology, CK levels, force deficit, and humoral immunity assessment. Protein and RNA expression analysis confirmed the successful AAV9-mediated delivery of transgenes to all target organs, including striated muscle, heart, and diaphragm (Fig. 3A,B). Transcript levels in TA muscle were comparable to those detected after direct intramuscular injection. Human codon-optimized $\mu$ Utrn was efficiently delivered to the sarcolemma, thus restoring the DAGC (Fig. 3C).

Morphological analysis of skeletal muscle, heart, and diaphragm showed myofiber damage and many centronucleated muscle cells, in agreement with the histopathological aspects of the disease. There were no prominent differences between treated and untreated $m d x$ mice. Features observed in all mice from both groups included areas of necrosis and regeneration as well as sites of inflammation and fibrosis, which are typical for DMD (Fig. 3D). There were no changes in other organs, except for the thyroid (Supplementary Fig. S2). One mouse exhibited a reduction in follicle number and hypertrophy of follicle epithelium 20 weeks after the injection of human codon-optimized $\mu U t r n$. We cannot definitively conclude whether these changes were related to rAAV9Hco- $\mu$ Utrn treatment or were individual age-related changes. It was previously shown that the systemic delivery of rAAV-based therapeutics can result in hypertrophy of the thyroid epithelium due to a reduction in thyroid hormone levels ${ }^{28}$.

Long-term functional assessment determined via hanging wire tests showed significant improvements in the group of $m d x$ mice treated with rAAV9-Hco- $\mu$ Utrn at several time points (Fig. 3E, weeks 6 and 17). Unexpectedly, on other weeks, functional improvements in the Hco- $\mu$ Utrn group were not significant, if any. In addition, at week 8, wild-type animals did not exhibit any difference from model mice due to unknown reasons (Fig. 3E). We suppose that the small number of animals in the experimental groups $(n=4)$ did not allow to exclude the influence of individual characteristics of animals and that greatly distorts the average value in the presence of outliers. Serum CK levels were markedly reduced to 6000-7000 in treated $m d x$ mice, as compared to approximately $9000 \mathrm{U} / \mathrm{L}$ in control $m d x$ mice (Fig. 3F). The specific force of isolated skeletal muscles from rAAV9-Hco- $\mu$ Utrn-injected mice was higher than that of untreated $m d x$. Similarly, moderate force drop improvements after repeated eccentric contractions were detected in treated animals, although not significant (Fig. 3G).

To assess the humoral immune response to long-term transgene expression, we detected Hco- $\mu$ Utrn-specific antibodies in the serum of treated animals. In parallel, we analyzed the sera of animals treated with the same dose of AAV9- $\mu$ Dys. We detected $\mu$ Dys-specific antibodies in sera from three of four AAV9-CMV- $\mu$ Dys-injected mice (Fig. 3H, №1, 2, 4 of the $\mu \mathrm{Dys}$ group). Antibody levels varied between samples, as indicated by the intensity of bands. The sera of wild-type (B10) and $m d x$ mice treated with DPBS did not contain transgene-specific antibodies. Hco- $\mu$ Utrn-specific antibodies were identified in sera from two out of four treated mice (Fig. 3H, №2, 3 of the $\mu$ Utrn group). However, band intensity was notably lower than for $\mu$ Dys-treated animals.

Taken together, the long-term monitoring of $m d x$ mice after intravenous injection of rAAV9-Hco- $\mu$ Utrn demonstrated persistent transgene expression in the skeletal muscle, heart, and diaphragm, with appropriate localization to the sarcolemma. Expression of Hco- $\mu$ Utrn allowed for functional recovery and protected muscle against contraction-induced damage. Further, the histopathological analysis did not reveal any toxicity effect associated with the expression of rAAV9-Hco- $\mu$ Utrn and vector administration at high doses. 

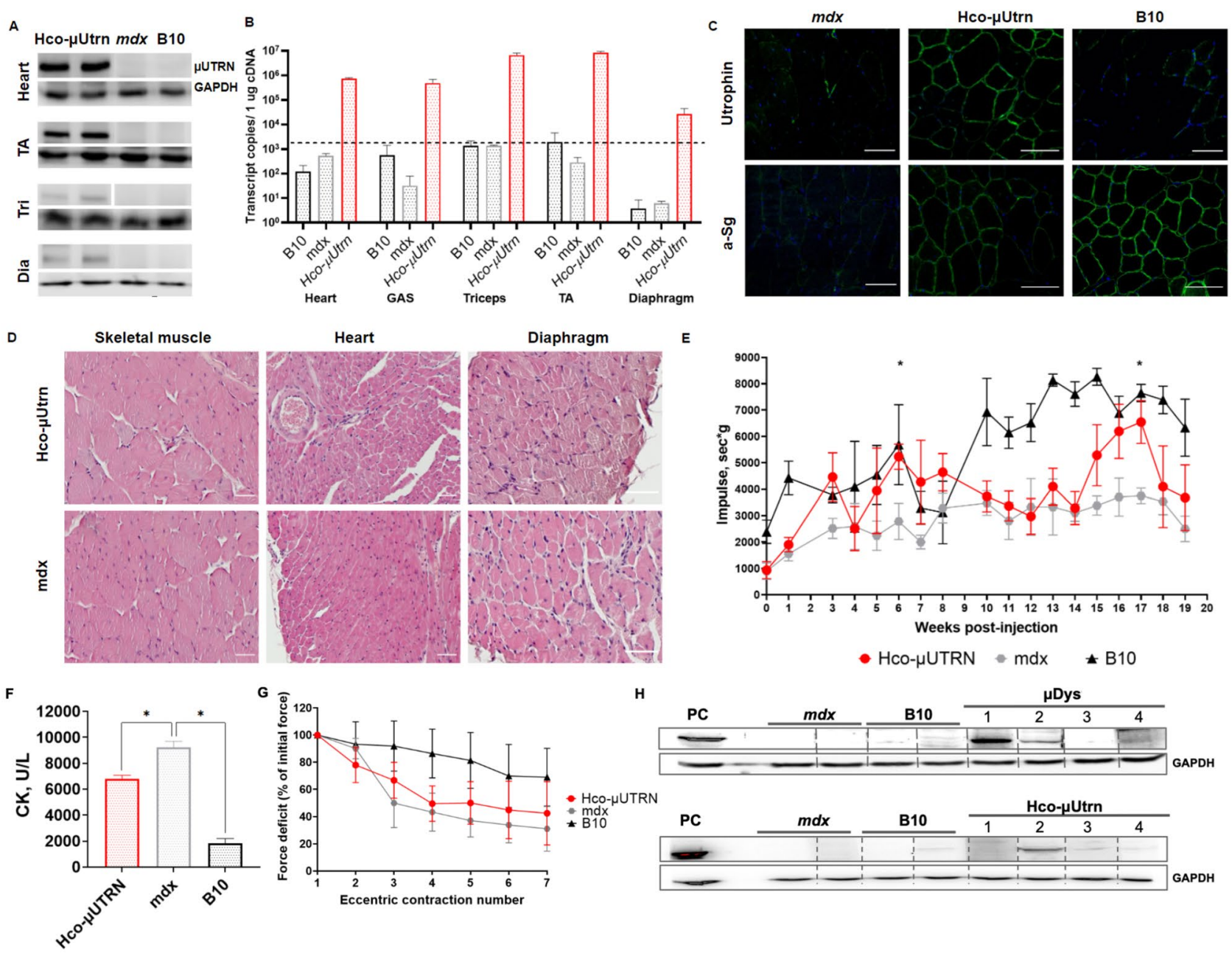

\section{E}
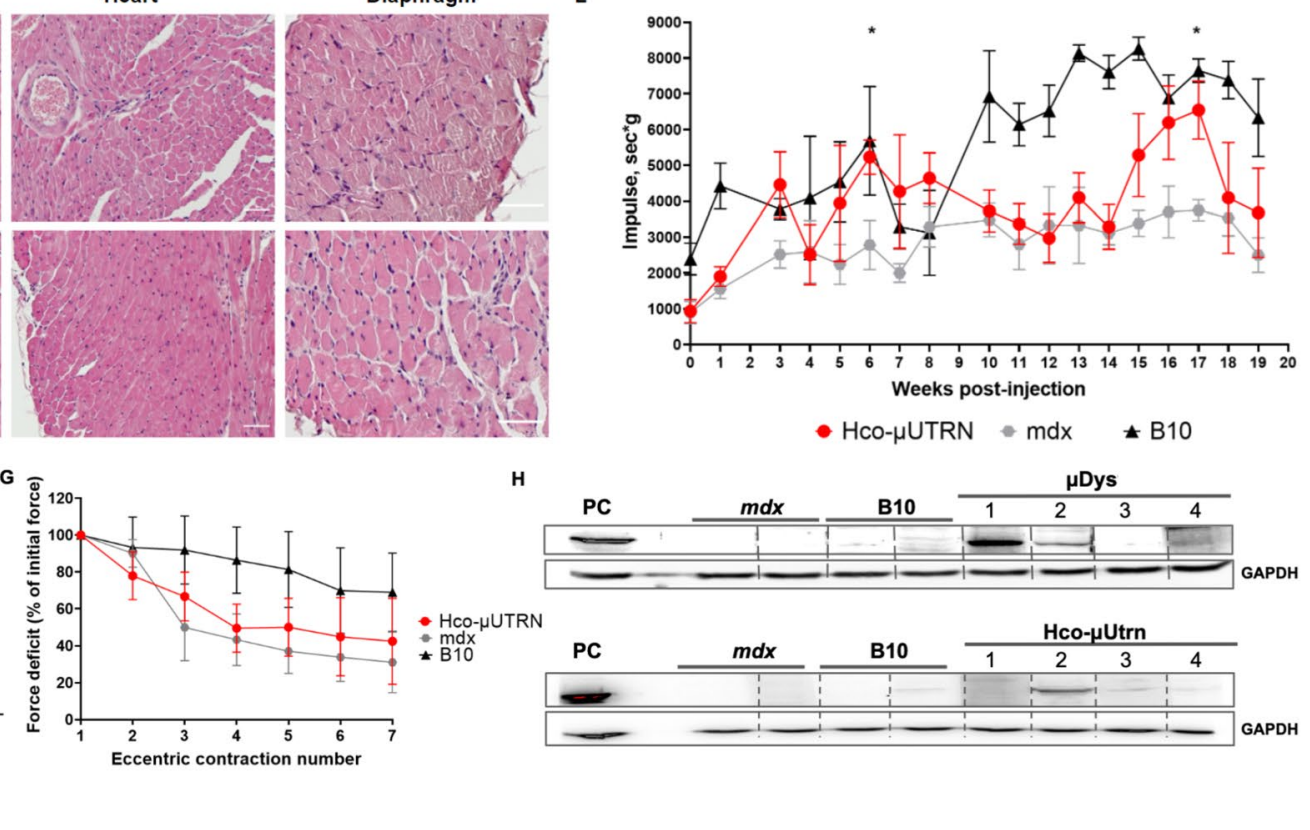

Figure 3. Systemic delivery of rAAV9-Hco- $\mu$ Utrn at dose $6 \times 10^{14} \mathrm{vg} \mathrm{kg}^{-1}$ for twenty-week studies in $m d x$ mice. (A) Western blot analysis of recombinant $\mu$ Utrn expression in the heart, tibialis anterior (TA), triceps (Tri), and diaphragm (Dia); loading control, GAPDH. Unprocessed full-length blots are presented in Supplementary Figure S10. (B) RT-qPCR analysis of Hco- $\mu$ Utrn expression in the heart, gastrocnemius (GAS), triceps, TA, and diaphragm. The dashed line represents the detection levels of negative controls. (C) Representative images of TA muscle cryosection immunofluorescence analysis after rAAV9-Hco- $\mu$ Utrn administration. Native and recombinant utrophin as well as $\alpha$-sarcoglycan were colored in green. Nuclei were counterstained with DAPI (blue). Scale bar, $100 \mu \mathrm{m}$. (D) Representative images of hematoxylin and eosin (H\&E)-stained skeletal muscle, heart, and diaphragm (see Fig. S2 for more organs). Scale bar, $100 \mu \mathrm{m}$. (E) Hanging wire test. The maximum hanging time of three trials during a 300-s wire test protocol normalized to mouse mass. (F) Creatine kinase (CK) levels in serum. (G) Percentage force drop following 20\% eccentric contraction of TA muscles of $m d x$ mice administered rAAV9-Hco- $\mu$ Utrn compared to those of vehicle control mice. (H) Western blot analysis of Hco$\mu U$ trn- and $\mu$ Dys-specific antibodies in the sera of treated mice (1:100 dilution). PC-positive control, sample incubated with antibodies against utrophin (Cau22354) and dystrophin (DysB); loading control, GAPDH. Unprocessed full-length blots are presented in Supplementary Figure S10. All values are presented as the mean \pm SEM $(n=4$ mice $)$, and statistical significance was set at $P<0.05$.

Intramuscular injection of rAAV9-Hco- $\mu$ Utrn-FLAG virus led to expression on the sarcolemma of TA muscles and functional improvements in $\mathbf{m d x}$ mice. To distinguish between native utrophin and recombinant $\mu$ Utrn during immunofluorescence, the FLAG epitope tag was used as a transgene marker. The addition of an $\mathrm{N}$-terminal FLAG tag to the $\mu$ Utrn sequence has been reported previously ${ }^{20,21}$. In the present study, the Hco- $\mu$ Utrn protein C-terminus was modified with the DYKDDDDK peptide sequence, allowing for detection of the transgene using antibodies against the utrophin N-terminus and the FLAG epitope. To determine whether the FLAG-tag had a negative impact on Hco- $\mu$ Utrn expression, localization, and functionality, we injected rAAV9-Hco- $\mu$ Utrn and rAAV9-Hco- $\mu$ Utrn-FLAG into the TA muscles of $m d x$ mice $\left(2 \times 10^{12} \mathrm{vg}\right.$ per TA muscle). Two weeks post-injection, transcript levels and function were analyzed. Hco- $\mu$ Utrn-FLAG as well as Hco- $\mu$ Utrn were successfully expressed on the sarcolemma of TA muscle cells (Fig. 4A, UTRN). Antibodies against the FLAG epitope recognized the Hco- $\mu$ Utrn-FLAG protein (Fig. 4A, FLAG). The expression level was similar for both transgenes (Fig. 4B). Further, both rAAV9-Hco- $\mu$ Utrn- and rAAV9-Hco- $\mu$ Utrn-FLAG-treated 

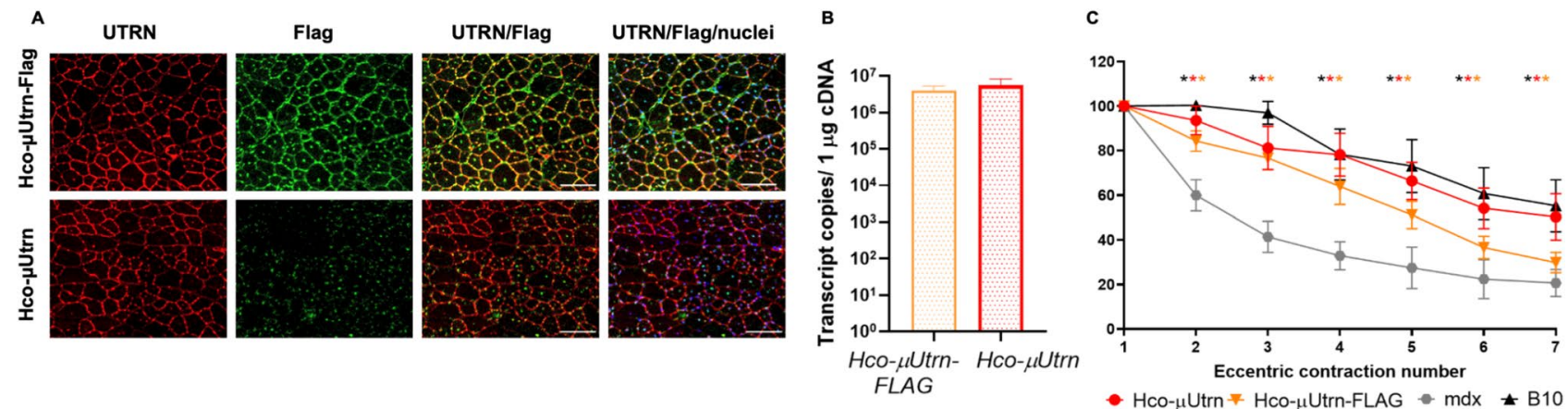

Figure 4. Addition of FLAG epitope to Hco- $\mu$ Utrn does not interfere with the expression, localization, and function of the recombinant protein after intramuscular administration at dose $2 \times 10^{12} \mathrm{vg} / \mathrm{TA}$ muscle. (A) Representative images of utrophin (red) and FLAG-epitope (green) immunofluorescence in TA muscles of $m d x$ mice after 2 weeks post rAAV9-Hco- $\mu$ Utrn and rAAV9-Hco- $\mu$ Utrn-FLAG administration. Nuclei were counterstained with Hoechst 33,342 (blue). Scale bar, $100 \mu \mathrm{m}$. (B) Comparison of $\mu$ Utrn and $\mu U t r n-F L A G$ expression level in treated $m d x$ TA muscles, as determined via RT-qPCR. Data are presented in transcript copies per $1 \mu \mathrm{g}$ cDNA. (C) Percentage force drop following $20 \%$ eccentric contractions of rAAV9-Hco- $\mu$ Utrn and rAAV9-Hco- $\mu$ Utrn-FLAG-treated $m d x$ TA muscle vs untreated $m d x$ and B10 mouse muscle. All values are presented as the mean \pm SEM $(n=8)$, and statistical significance was set at $P \leq 0.05$.

$m d x$ TA muscles exhibited a statistically significant improvement in contractile function when compared to untreated $m d x$ mouse muscles (Fig. 4C). Thus, FLAG-tagged Hco- $\mu$ Utrn can be used for further experiments in $m d x$ mice or other animals with endogenous utrophin expression.

High-dose rAAV9-Hco- $\mu$ Utrn-FLAG administration does not cause toxicity in rats. In order to assess the toxicity of human codon-optimized $\mu$ Utrn delivered via the rAAV9 vector, we administered rAAV9Hco- $\mu$ Utrn-FLAG to male rats through intravenous injection. Low $\left(2 \times 10^{14} \mathrm{vg} \mathrm{kg}^{-1}\right)$ and high $\left(6 \times 10^{14} \mathrm{vg} \mathrm{kg}^{-1}\right)$ doses were tested. Weaning rats were injected at 3 weeks of age, followed by extensive daily monitoring. Acute and subacute toxicity evaluation was performed on days 3 and 14, respectively (Fig. 5A).

All animals survived the duration of the study, with no significant changes in clinical signs, organ weights, histopathological findings, hematological and coagulation parameters, as well as urine biochemistry. Biochemical blood tests indicated that the levels of ALT, AST, ALP, and GGT did not differ between treated and control rats (Table 1). There was a significant increase in creatinine levels on day 3 in the group receiving a low dose (Table 1), but there was no difference on day 14 . The groups did not differ in mass and total activity, as determined via the open field test (Fig. 5D). Daily monitoring of animals confirmed no changes in the condition of the skin, hair, and visible mucous membranes. Ophthalmoscopic examination, ECG recording, urine biochemistry and Irwin tests also confirmed the lack of toxicity associated with rAAV9-Hco- $\mu$ Utrn-FLAG (Supplementary Fig. S5-S7).

At the two-week time point, several organs from the treated animals were subjected to transgene expression analysis. The Hco- $\mu$ Utrn transcript was detected in the diaphragm, heart, TA, gastrocnemius (GAS), as well as the liver, thus confirming successful rAAV9-mediated delivery and efficient expression (Fig. 5B). Dose-dependent expression was evident in all analyzed organs, except for the diaphragm. The high level of transgene transcripts in the liver can be explained by the presence of the constitutive CMV promoter in the transgene construct.

Taken together, our toxicity study confirmed that neither the rAAV9-Hco- $\mu$ Utrn-FLAG vector itself nor Hco- $\mu$ Utrn expression exhibited toxicity in rats.

Muscle-specific SPC5-12 and MHCK7 promoters drive $\mu$ Utrn expression and ensure force improvement after intramuscular injection in $\mathbf{m d x}$ mice. To further reduce immunogenicity, we decided to test an $\mathrm{Hco}-\mu U$ trn construct under muscle-specific promoters. To this end, we replaced the constitutive CMV promoter with MHCK7 and SPc5-12 promoters $^{29,30}$. In contrast to previous reports ${ }^{20}$, including a SPc5-12 promoter in the $\mu$ Utrn-expressing construct did not lead to the reduction of AAV vector yield (Supplementary Table S8). The modified constructs were administered to 7 -week-old $m d x$ mice via intramuscular injection at a dose of $2 \times 10^{12} \mathrm{vg}$ per TA muscle.

We confirmed the induction of $\mu$ Utrn expression by all constructs via western blot analysis and immunostaining at day 14 following injection (Fig. 6A,D). Protein levels from constructs under CMV, MHCK7, and SPc5-12 promoters did not differ significantly between each other (Fig. 6B). However, the SPc5-12 promoter induced three-fold lower mRNA levels relative to CMV and MHCK7, as determined via RT-qPCR analysis (Fig. 6C). Restoration of DAGC on the sarcolemma was achieved in all groups, although highest $\alpha 1$-syntrophin expression was observed in the rAAV9-CMV-Hco- $\mu$ Utrn-treated group (Fig. 6D).

We observed the improvement of tetanic force in all treated TA muscles when compared to the muscles of untreated $m d x$ mice. All rAAV9-Promoter- $\mu$ Utrn variants led to a similar force deficit after eccentric contractions (Fig. 6E). The SPc5-12 promoter group exhibited the best specific force relative to those in the CMV and MHCK7 groups, which was also comparable to the force of wild-type controls (Fig. 6F). Thus, muscle-specific promoters 


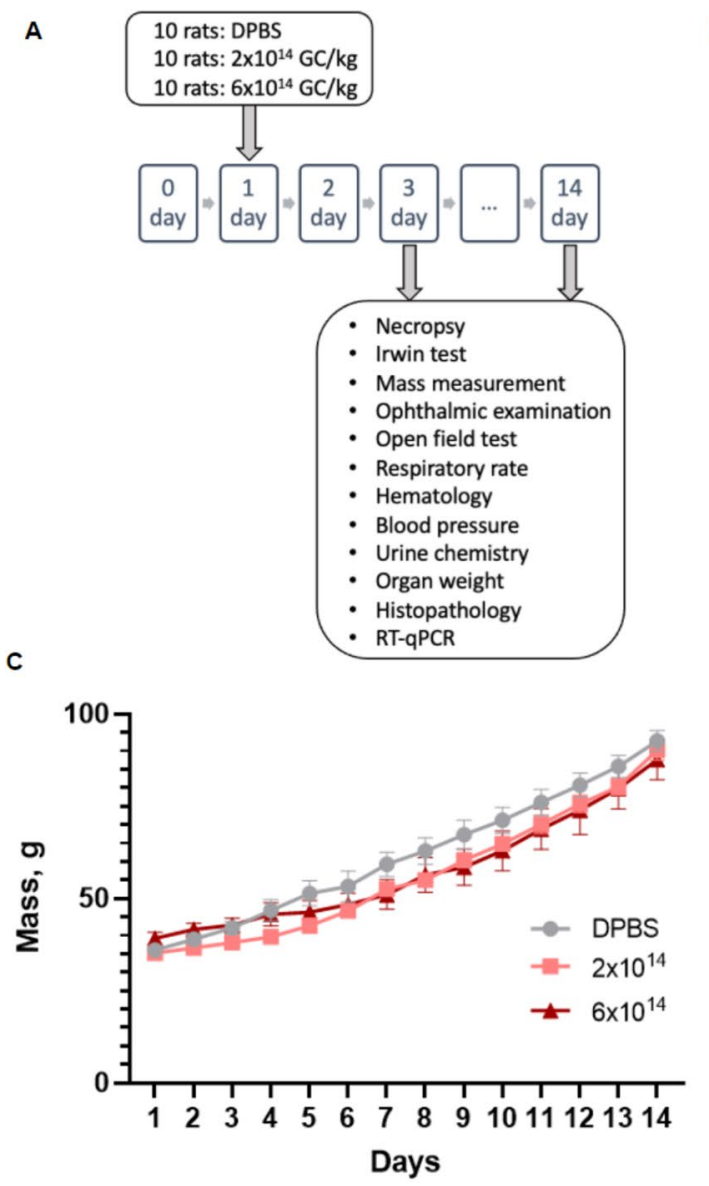

B
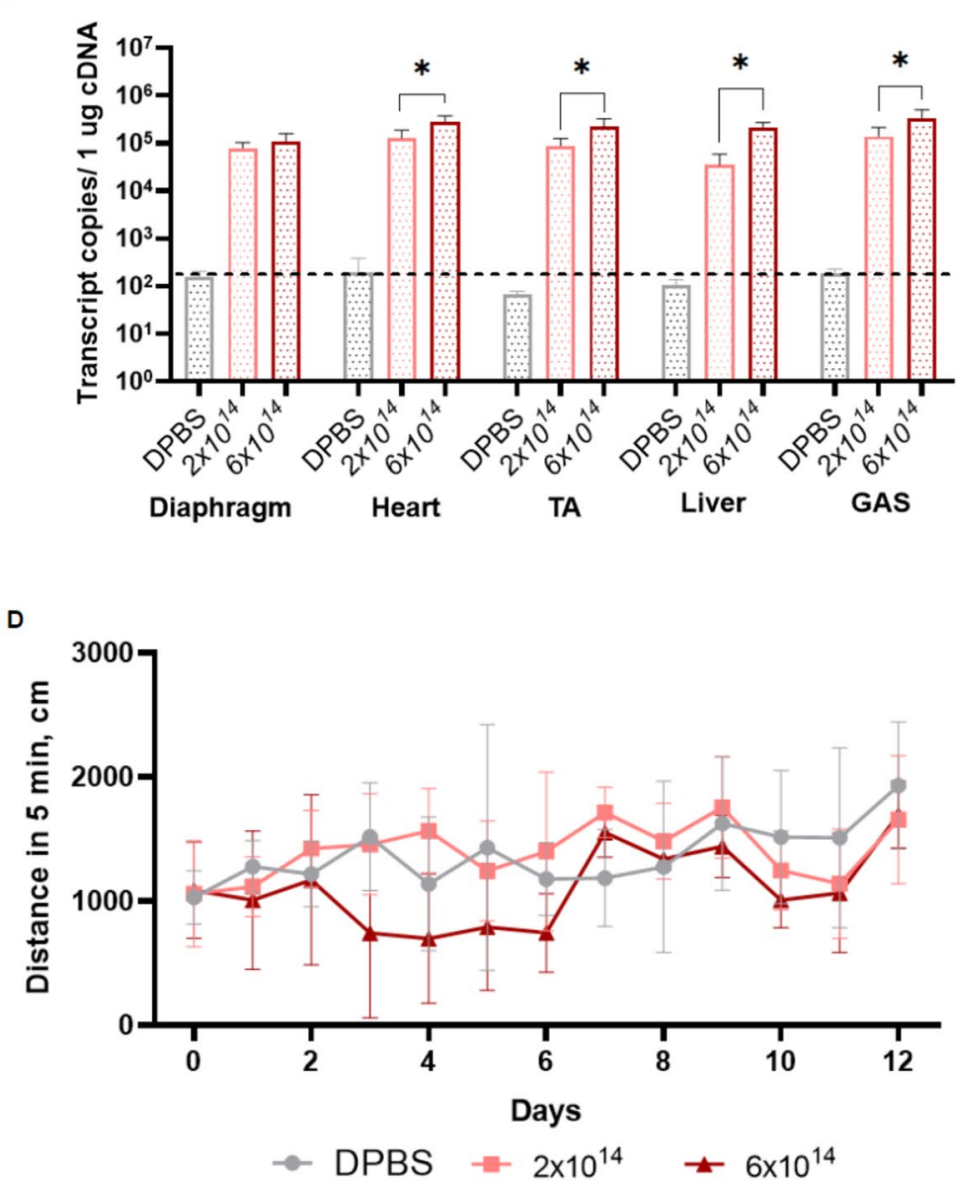

Figure 5. Systemic administration of rAAV9-Hco- $\mu$ Utrn-FLAG at doses $2 \times 10^{12}$ and $6 \times 10^{12} \mathrm{vg} \mathrm{kg}^{-1}$ does not cause toxicity in rats after 3 and 14 days post injection. (A) Study design. (B) Transgene expression levels in the diaphragm, heart, tibialis anterior (TA), liver, and gastrocnemius (GAS) of rats, as determined via RT-qPCR. Values are expressed as transcript copies per $1 \mu \mathrm{g}$ cDNA. The dashed line represents the detection levels of negative controls. (C) Body weight changes in experimental and control animals. (D) Distance traveled in 5 min during the open field test. All values are presented as the mean \pm SEM ( $n=5 /$ time point). Statistical significance was set at $P \leq 0.05$.

\begin{tabular}{|c|c|c|c|c|c|c|}
\hline \multirow[b]{2}{*}{ Day, group } & \multicolumn{3}{|l|}{ Day 3} & \multicolumn{3}{|l|}{ Day 14} \\
\hline & DPBS & $2 \times 10^{14} \mathrm{vg} \mathrm{kg}^{-1}$ & $6 \times 10^{14} \mathrm{vg} \mathrm{kg}^{-1}$ & DPBS & $2 \times 10^{14} \mathrm{vg} \mathrm{kg}^{-1}$ & $6 \times 10^{14} \mathrm{vg} \mathrm{kg}^{-1}$ \\
\hline Albumin, $g / \mathrm{L}$ & $27.0 \pm 4.6$ & $29.4 \pm 2.9$ & $25.4 \pm 20,0$ & $28.8 \pm 2.3$ & $27.8 \pm 3.1$ & $26.8 \pm 2.2$ \\
\hline Total protein, $\mathrm{g} / \mathrm{L}$ & $38.2 \pm 5.7$ & $35.6 \pm 2.7$ & $40.8 \pm 1.8$ & $44.4 \pm 4.3$ & $43.9 \pm 4.9$ & $39.1 \pm 3.1$ \\
\hline Creatinine, $\mu \mathrm{mol} / \mathrm{L}$ & $25.9 \pm 2.1$ & $34.2 \pm 3.6 \#$ & $29.6 \pm 1.6 \dagger$ & $30.2 \pm 3.1$ & $29.2 \pm 4.7$ & $30.3 \pm 4.0$ \\
\hline Total cholesterol, $\mathrm{mmol} / \mathrm{L}$ & $2.3 \pm 0.3$ & $2.1 \pm 0.2$ & $2.1 \pm 0.3$ & $1.9 \pm 0.1$ & $2.1 \pm 0.2$ & $2.0 \pm 0.2$ \\
\hline Glucose, $\mathrm{mmol} / \mathrm{L}$ & $8.8 \pm 0.8$ & $8.3 \pm 0.8$ & $8.3 \pm 1.0$ & $9.9 \pm 3.2$ & $10.6 \pm 1.7$ & $8.1 \pm 1.8$ \\
\hline ALT, IU/L & $64.3 \pm 9.7$ & $64.6 \pm 13.3$ & $57.6 \pm 16.5$ & $79.1 \pm 12.6$ & $71.1 \pm 11.4$ & $80.2 \pm 9.7$ \\
\hline AST, IU/L & $154.9 \pm 48.7$ & $227.5 \pm 50.6$ & $210.2 \pm 50.6$ & $201.0 \pm 86.4$ & $184.1 \pm 71.7$ & $150.7 \pm 26.1$ \\
\hline GGT, IU/L & $0.3 \pm 0.6$ & $0.1 \pm 0.1$ & $0.1 \pm 0.1$ & $1.0 \pm 0.9$ & $0.8 \pm 0.9$ & $0.3 \pm 0.3$ \\
\hline ALP, IU/L & $716.1 \pm 148.6$ & $633.4 \pm 139.4$ & $657.6 \pm 166.9$ & $648,0 \pm 180.7$ & $677.1 \pm 127.5$ & $673.3 \pm 176.8$ \\
\hline $\mathrm{K}^{+}, \mathrm{mmol} / \mathrm{L}$ & $5.2 \pm 0.6$ & $6.0 \pm 1.1$ & $5.9 \pm 0.7$ & $5.6 \pm 1.6$ & $5.5 \pm 1.3$ & $4.5 \pm 0.5$ \\
\hline $\mathrm{Na}^{+}, \mathrm{mmol} / \mathrm{L}$ & $142.8 \pm 4.4$ & $147.5 \pm 7.5$ & $143.0 \pm 3.6$ & $143.7 \pm 3.9$ & $146.5 \pm 10.0$ & $142.5 \pm 1.8$ \\
\hline
\end{tabular}

Table 1. Results of blood biochemical test in rats on days 3 and 14 after a single intravenous injection of rAAV9-Hco- $\mu$ Utrn-FLAG. All data are presented as mean \pm SD. $\# P=0.0007$ when compared to the control group (DPBS, day 3 ). $\dagger P=0.0380$ when compared to the group treated with a low dose of rAAV9-Hco- $\mu$ UtrnFLAG $\left(2 \times 10^{14} \mathrm{vg} \mathrm{kg}^{-1}\right.$, day 3$)$. In both cases (\# and $\left.\dagger\right)$, statistical analysis was performed via ANOVA and Tukey's post-hoc test. 


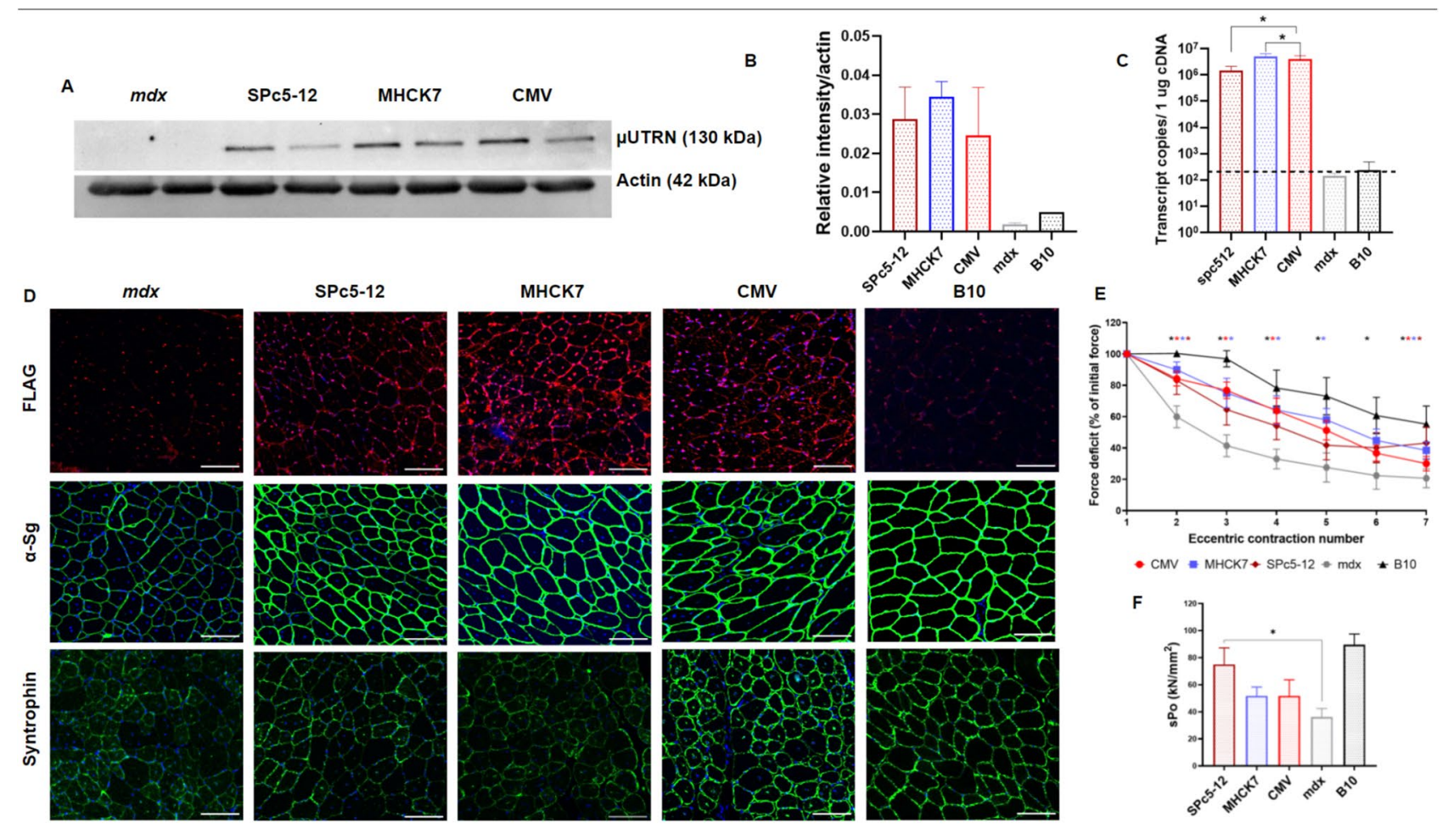

Figure 6. Muscle-specific SPc5-12 and MHCK7 promoters drive $\mu$ Utrn expression and ensure force improvement after intramuscular injection at dose $2 \times 10^{12} \mathrm{vg} / \mathrm{TA}$ in $m d x$ mice. (A) Western blot analysis of recombinant $\mu$ Utrn expression in TA muscles after 2 weeks post injection, with $\alpha$-actin as the loading control. Unprocessed full-length blots are presented in Supplementary Figure S10. (B) Hco- $\mu$ Utrn protein levels in rAAV9-Promoter-Hco- $\mu$ Utrn-treated $m d x$ TA muscles determined via western blot and normalized to $\alpha$-actin levels. (C) Analysis of $\mu$ Utrn transgene expression via RT-qPCR. Values are expressed as transcript copies per $1 \mu \mathrm{g}$ cDNA. The dashed line represents the detection levels of negative controls. (D) Representative images of $\mu$ Utrn-FLAG (FLAG), $\alpha$-sarcoglycan (a-Sg), and $\alpha 1$-syntrophin immunofluorescence in TA muscle crosssections. Nuclei were counterstained with Hoechst 33,342 (blue). Scale bars, $100 \mu \mathrm{m}$. (E) Percentage force drop following 20\% eccentric contraction and (F) specific force of TA muscles from $m d x$ mice administered rAAV9$\mu U t r n-F L A G$ compared to those from vehicle control mice. Maximal isometric force and cross-sectional area of TA muscles are present in Supplementary Figure S11. All values are presented as the mean \pm SEM $(\mathrm{N}=8$ muscles), and statistical significance was set at $P \leq 0.05$.

MHCK7 and SPc5-12 were as effective as the constitutive CMV promoter. Further, all tested promoters induced Hco- $\mu$ Utrn expression and functional improvement at comparable levels.

\section{Discussion}

Studies utilizing microdystrophin vectors have previously demonstrated that codon optimization can significantly increase the level of transgene protein ${ }^{22,23}$. The current work focused on $\mu$ Utrn codon optimization in an attempt to enhance gene expression in the skeletal muscles and heart, thereby contributing to functional improvement following rAAV9-mediated gene transfer. We used the codon bias of the most prevalent muscle proteins, namely actin and myosin (see Supplementary Fig. S1 for details), and optimized $63 \%$ of the codons in human $\mu$ Utrn. It should be noted that although the $\mu$ Utrn cDNAs were optimized for use in humans, codon usage and transfer RNA frequencies are similar across vertebrates ${ }^{31}$, which suggests that cDNAs were appropriate for the mouse model used in our study. The resulting human codon-optimized $\mu$ Utrn coding sequence was compared to a previously described murine codon-optimized $\mu$ Utrn sequence ${ }^{17}$ and a control non-optimized human sequence ${ }^{32}$ with the same domain structure. A similar strategy was followed by Song and colleagues ${ }^{20}$ for both human and canine sequences. In addition to codon-optimization, they focused on $\mu$ Utrn structure and chose internal deletion in order to avoid disruption of the native triple helical repeat within the Rod-domain.

In the present work, codon optimization of human $\mu$ Utrn resulted in high levels of $\mu$ Utrn expression in TA muscles following intramuscular administration of $2 \times 10^{12} \mathrm{vg}$ rAAV9. Protein expression from Hco- $\mu$ Utrn exceeded that from $\mathrm{H}-\mu \mathrm{Utrn}$ (Fig. 2A,B) and protected muscle against contraction-induced injury (Fig. 2F). Unexpectedly, TA muscles of $m d x$ mice, treated with rAAV9-H- $\mu$ Utrn, exhibited the most pronounced improvement in specific force (Fig. $2 \mathrm{G}$ ).

Original murine $\mu$ Utrn was previously tested in $m d x: u t r n^{-/-}$mice using an rAAV6 vector for gene delivery ${ }^{17}$. After the systemic administration of rAAV6- $\mu$ Utrn $\left(3 \times 10^{12} \mathrm{vg} /\right.$ mouse $)$ to 4 -week-old $m d x$ : utrn ${ }^{-/-}$mice, authors confirmed alleviation of the dystrophic phenotype and increased force production. In similar experimental conditions $\left(1.2 \times 10^{13} \mathrm{vg} /\right.$ mice, 6 -week-old $m d x$ mice), we detected high $\mu$ Utrn expression levels in striated muscles, 
heart, diaphragm, and functional improvement at several time points within 20 weeks after the injection of rAAV9-Hco- $\mu$ Utrn. However, using small animal numbers for functional tests doesn't allow detection of significant changes throughout the experiment and should be considered as a limitation of present study. Further, no toxicity was observed following systemic vector delivery. Treatment also lowered serum CK level, improved the specific force of isolated TA muscle, and protected against contraction-induced injury (Fig. 2). In a recent study Banks et al. ${ }^{21}$ demonstrated that the number of $\mu$ Utrn-positive myofibrils depends on the experiment duration. They observed high levels of $\mu$ Utrn expression two weeks after the intravenous infusion of rAAV6-CK8e-Flag$\mu$ Utrn to $\mathrm{mdx}^{4 \mathrm{cv}}$ mice, which was markedly reduced at later time points. Considering that $\mu$ Utrn co-exists with full-length utrophin at the sarcolemma and the latter is found at higher levels in type $1 \mathrm{a}, 2 \mathrm{a}$, and $2 \mathrm{~d}$ fibers ${ }^{21}$, which are less protected by $\mu$ Utrn, their results suggested that endogenous utrophin expression may adversely impact the therapeutic potential of $\mu$ Utrn. In the present study, we detected similar mRNA levels in TA muscle after direct intramuscular and intravenous injection of rAAV9-CMV- $\mu$ Utrn into $m d x$ mice expressing fulllength utrophin (Figs. 2C and 3B). Both injections protected muscle from force drop during repeated eccentric construction, and short-term analysis revealed more pronounced functional improvement (Fig. 2F-2 weeks and $3 \mathrm{~g}-20$ weeks), which indirectly confirms the findings of Banks and colleagues.

Safety evaluation of novel gene therapy vectors in wild-type animals is an essential step in the development of these therapeutics ${ }^{2}$. Herein, we tested FLAG-modified Hco- $\mu$ Utrn in wild-type rats and did not observe any adverse effects after intravenous injections of $2 \times 10^{14} \mathrm{vg} \mathrm{kg}^{-1}$ and $6 \times 10^{14} \mathrm{vg} \mathrm{kg}^{-1}$ at time points corresponding to acute and subchronic toxicity (Fig. 5, Table 1, and Supplementary Fig. S5-S7). Hco- $\mu$ Utrn was successfully delivered and expressed in striated muscle, heart, diaphragm, and the liver (Fig. 5B). Taking into consideration that a high dose of administered rAAV9-Hco- $\mu$ Utrn harboring the CMV promoter might lead to some transgene expression in non-target tissues, $\mu$ Utrn gene therapy can be considered extremely safe. However, further studies on the long-term effects of $\mu$ Utrn expression and safety evaluation in large animals will add to existing evidence in favor of the safety of this therapeutic approach ${ }^{33-35}$.

As previously mentioned, the generation of immune responses against the foreign dystrophin protein represents a major concern in dystrophin replacement therapy. An immune response against microdystrophin following the first intramuscular vector injection was previously confirmed in human patients receiving AAV $2.5^{3}$. Various immune reactions have also been observed in other clinical trials evaluating systemic rAAV-based delivery of microdystrophin ${ }^{2,36}$. In one trial (NCT03368742), two patients treated with high $\left(2 \times 10^{14} \mathrm{vg} \mathrm{kg}^{-1}\right)$ and low $\left(5 \times 10^{13} \mathrm{vg} \mathrm{kg}^{-1}\right)$ doses exhibited activation of the complement system with signs of cardiopulmonary decline, although all transient serious adverse events were fully resolved. In another clinical trial (NCT03362502), immune responses measured by T-cell responses on ELISPOT and neutralizing antibody levels were documented in all participants ${ }^{37}$. The great advantage of utrophin is its non-immunogenic nature, achieved through expression in the thymus during the early stages of development ${ }^{38}$. The lower immunogenicity of utrophin was first demonstrated in a study comparing miniutrophin and minidystrophin delivery using an adenoviral vector ${ }^{39}$. In a recent study, Song et al. ${ }^{20}$ compared the immunogenicity of microdystrophin and $\mu$ Utrn, confirming this notion. In the current work, we also demonstrated the lower immunogenicity of $\mu$ Utrn constructs over $\mu D y s$. Further, we investigated the humoral and cellular immune responses against $\mu$ Utrn and $\mu$ Dys proteins. As expected, $\mathrm{M}-\mu \mathrm{Utrn}$ delivery results in lower CD8 + CTL infiltration rates than other transgene products in the muscles of $m d x$ mice. Hco- $\mu$ Utrn-treated muscles also showed lower infiltrating CTL number relative to non-treated $m d x$ and $\mu$ Dys groups. The presence of transgene-specific antibodies in the sera of $m d x$ mice during long-term monitoring also confirmed the advantage of $\mu$ Utrn over $\mu$ Dys, although the sample size was low $(n=4)$, which is a significant limitation of the study.

Ubiquitous promoters, such as CMV, RSV, and EF1a, provide strong and robust transgene expression but may trigger potent immune responses in patients ${ }^{3}$. Thus, current clinical trials of microdystrophin delivery evaluate constructs under muscle-specific promoters $\mathrm{CK}^{40}, \mathrm{MHCK}^{29}$ and $\mathrm{MCK}^{41}$. Thus, the latest research on AAV-mediated microutrophin delivery has also focused on the usage of muscle-specific promoters ${ }^{20,21}$. In the present study, the CMV promoter facilitated high $\mathrm{Hco}-\mu \mathrm{Utrn}$ expression in the rat liver, comparable to levels in muscle tissue (Fig. 4B) although without any toxicity after systemic AAV9-CMV-Hco- $\mu$ Utrn-FLAG delivery. We decided to compare the constitutive CMV promoter with rationally designed muscle-specific natural promoter MHCK $7^{29}$ and synthetic promoter SPc5-12 $2^{30}$. Following intramuscular injection, muscle-specific promoters demonstrated high activity, comparable to that of the CMV promoter. All analyzed promoters ensured sufficient therapeutic protein expression, resulting in restoration of the DAGC as well as functional recovery. For a more comprehensive comparison, better examination of functional changes and potential toxicity, we plan to extend our data with systemic delivery of muscle-specific promoters-driven $\mu$ Utrn. Although the lack of these experiments is a limitation of the present study, we want to note that the possible off-target effects are expected to be higher when using a constitutive promoter such as CMV promoter. However, we did not observe any toxicity in histopathology, hematology, blood and urine biochemistry and other parameters after high-dose AAV9-CMVHco- $\mu$ Utrn-FLAG delivery studies on rats (Supplementary Fig. S5-S7).

Taken together our results demonstrated the potential of novel human codon-optimized $\mu$ Utrn delivered via rAAV9 as gene therapy for DMD. Short-term evaluation of intramuscular delivery as well as long-term studies of systemic administration in $m d x$ mice confirmed transgene functionality. Further, extensive toxicity studies in wild-type rats confirmed the safety of our gene therapy. We also demonstrated that muscle-specific promoters MHCK7 and SPc5-12 drove sufficient rAAV9- $\mu$ Utrn expression for the amelioration of the dystrophic phenotype in $m d x$ mice. The current results establish a basis for the further development of human codon-optimized $\mu$ Utrn combined with muscle-specific promoters as a safe and efficient gene therapy for DMD. 


\section{Materials and methods}

Ethics. All animal procedures were performed in accordance with Directive 2010/63/EU is the European Union (EU) legislation "on the protection of animals used for scientific purposes" and Animal Research: Reporting of In Vivo Experiments (ARRIVE) guidelines and regulations. Experiments were approved by the Local Ethics Committees of Institute of Gene Biology and Belgorod State National Research University.

$\mu U t r n$ design and DNA constructs. To design human $\mu$ Utrn with a $\Delta \mathrm{R} 4-\mathrm{R} 21 / \Delta \mathrm{CT}$ domain structure, the full-length human utrophin protein sequence (UniProtKB-P46939) was aligned to the murine $\mu$ Utrn protein (GenBank: ABY20737.1) previously reported by Odom et al. ${ }^{17}$ Protein segments corresponding to 1-685aa, 2690-3165aa, 3431-3433aa in human utrophin were selected, and human $\mu$ Utrn cDNA $(H-\mu U t r n)$ was created. The nucleotide sequence of H- $\mu$ Utrn was then codon-optimized (Hco- $\mu$ Utrn, GenBank: OK094718) based on the codon usage in skeletal muscle and cardiac cells (actin, myosin). Details of the codon optimization procedure are provided in the Supplemental Information. All $\mu$ Utrn cDNAs were modified to include a consensus Kozak sequence. cDNA synthesis was carried out by Shinegene Bio-Technologies, Inc. (Shanghai, China). Transgenes were cloned into a pAAV-MCS vector (Agilent Technologies), and the engineered plasmids were designated as pAAV-CMV-M- $\mu$ Utrn, pAAV-CMV-H- $\mu$ Utrn, and pAAV-CMV-Hco- $\mu$ Utrn, respectively. To generate pAAVCMV-Hco- $\mu$ Utrn-FLAG, human codon-optimized $\mu U t r n$ was fused with a C-terminal FLAG epitope via PCR using the GenPak ${ }^{\mathrm{Tx}}$ PCR kit (Russia) with the following primers: Hco- $\mu$ Utrn-FseI-F 5'-tgcgtggacatgtgcctg-3' and mi-C-Flag-R 5'-ATGgaattcTTACTTGTCGTCATCGTCTTTGTAGTCcatggectgggtctccaggtt-3'. In pAAVMHCK7-Hco- $\mu$ Utrn-FLAG and pAAV-SPc5-12-Hco- $\mu$ Utrn-FLAG constructs, promoters MHCK7 and SPc5-12 were cloned under MluI/SacI restriction sites, replacing the CMV promoter in pAAV-CMV-Hco- $\mu$ Utrn-FLAG.

rAAV9 vector production. rAAV9 encoding $\mu U t r n$ and $\mu D y s$ were produced via the triple transfection method as previously described ${ }^{42}$. Briefly, adherent HEK293T (ATCC) cells were maintained in high-glucose DMEM (Gibco, USA) supplemented with $10 \%$ v/v fetal bovine serum (FBS, Biosera Europe, France) and PenStrep. Transfection was performed using linear PEI (polyethyleneimine) (Polysciences, USA) and the mix of plasmids for virus assembly: (i) pAAV- $\mu$ Utrn or pAAV- $\mu$ Dys expression vectors, (ii) rep2-cap9-modified AAV plasmid (Penn Vector Core, USA), and (iii) pHelper (Agilent, USA). Seventy-two h after transfection, the cells were lysed with $0,5 \% \mathrm{v} / \mathrm{v}$ Triton X-100, $5 \mu \mathrm{g} \mathrm{mL}^{-1}$ RNAse A, and $10 \mu \mathrm{g} \mathrm{mL} \mathrm{m}^{-1}$ DNAse A (Calbiochem, USA) for $1 \mathrm{~h}$ at $37^{\circ} \mathrm{C}$ with shaking. Clarified and concentrated virus suspension was loaded on a modified version of an iodixanol (Sigma Aldrich, USA) density step gradient $(60 \%, 40 \% \text {, and } 25 \%)^{43}$ and subjected to ultracentrifugation for $1 \mathrm{~h}$ at $350000 \mathrm{~g}$ and $18{ }^{\circ} \mathrm{C}$. The AAV-containing fraction was dialyzed using a $100 \mathrm{kDa}$ membrane against the storage buffer (PBS, $350 \mathrm{mM} \mathrm{NaCl}, 0.1 \%$ Pluronic F-68 (Gibco), and the purified suspension was further concentrated and sterilized ${ }^{44}$. A quantitative PCR-based method was used to determine the encapsidated vector genome ( $\mathrm{vg}$ ) titer using primers and a fluorescence probe targeting ITR sequences ${ }^{45}$. Absence of protein impurities in preparations was confirmed via Silver staining (ThermoFisher, USA) (Supplementary Fig. S9).

rAAV9 administration and tissue collection in mice. Dystrophin-deficient C57BL/10ScSn-Dmd ${ }^{\mathrm{mdx}} / \mathrm{J}$ $(m d x)$ male mice and C57BL/10ScSnJ (B10, parental strain) male mice were obtained from Jackson Laboratory. Before AAV- $\mu$ Utrn administration, mice were anesthetized with tiletamine hydrochloride/zolazepam hydrochloride mix $(50 \mathrm{mg} / \mathrm{kg})$ and xylazine $(5 \mathrm{mg} / \mathrm{ml})$ via intraperitoneal injections. For intramuscular delivery $(\mathrm{i} / \mathrm{m})$, $m d x$ mice received injections of AAV- $\mu$ Utrn diluted in $40 \mu$ DPBS into each TA muscle $\left(2 \times 10^{12} \mathrm{vg} / \mathrm{muscle}\right)$. For intravenous delivery (i/v), $m d x$ mice received injections of AAV- $\mu$ Utrn diluted in $300 \mu \mathrm{LPBS}$ into the retroorbital venous sinus $\left(150 \mu \mathrm{l}\right.$ per each sinus, $\left.6 \times 10^{14} \mathrm{vg} \mathrm{kg}^{-1}\right)$. Control mice received injections of DPBS in the corresponding volume.

Mice were euthanized at 2 weeks post-injection for $\mathrm{i} / \mathrm{m}$ delivery or 20 weeks for $\mathrm{i} / \mathrm{v}$ delivery, after terminal physiological tests. Muscles were collected for protein, RNA, and immunofluorescence analysis. Muscles for protein and RNA extraction were snap-frozen in liquid nitrogen, while tissues for staining were embedded in mounting medium Tissue-Tek OCT Compound (Sakura Finetek, Japan) and frozen in isopentane (PanReac, Spain) precooled with liquid nitrogen. All samples were stored at $-70^{\circ} \mathrm{C}$ before analysis.

CK. Blood was collected from jugular veins before euthanasia. Blood was left for $15 \mathrm{~min}$ at room temperature and centrifuged ( $3500 \mathrm{~g}$ for $10 \mathrm{~min}$ at $4{ }^{\circ} \mathrm{C}$ ) to promote clot formation and serum collection. CK activity was determined using the Creatine Kinase Activity Assay Kit (Sigma-Aldrich) and the Synergy 4 instrument (BioTek Instruments, USA).

Histopathology. Organs were weighed and fixed in $10 \%$ buffered formalin and embedded in paraffin. $5-\mu \mathrm{m}$-thick transverse sections were stained with $\mathrm{H} \& \mathrm{E}$ according to routine procedure described in Slaoui et al. ${ }^{46}$. Images of H\&E-stained sections were acquired with a Zeiss Axiocam camera at $200 \times$ magnification.

Toxicity assessment. Toxicity studies were conducted at Belgorod State University (Russia). The study included 30 male Wistar rats (21 days old). rAAV9-Hco- $\mu$ Utrn-FLAG was tested at two doses: $2 \times 10^{14} \mathrm{vg} \mathrm{kg}^{-1}$ and $6 \times 10^{14} \mathrm{vg} \mathrm{kg}^{-1}$ after single injection via intravenous route (lateral tail vein). Euthanasia was performed by exsanguination with cardiac puncture under isoflurane inhalation. Animal behavior in the open field test was recorded daily using an ActiTrack system (Panlab, Spain). A modified Irwin test was performed daily for general assessment of the animals welfare. Terminal procedures, consisting of electrocardiography (ECG), blood pressure measurement, ophthalmoscopy, collection of whole blood, serum, and urine samples as well as necropsy 
were performed on the days 3 ( $1 / 2$ animals) and 14 ( $1 / 2$ animals). Collected urine was analyzed using test strips and a semi-automatic urine analyzer Urit 180 Vet (URIT Medical Electronic Group, China). Complete blood count analysis was performed with use of automatic hematology analyzer CELL-DYN 3700 (Abbott Diagnostics, USA). Biochemical blood analysis was performed on an A25 analyzer (Biosystems, Spain) using reagents and control materials from Hospitex Diagnostics. ECG was performed using the ECG-1003 DIXION machine (Dixon Technologies, India).

Immunofluorescence. Transverse $10-\mu \mathrm{m}$-thick sections of muscles were obtained using a Leica CM 1510-1 cryostat. Muscle sections were fixed with 4\% paraformaldehyde (AppliChem, Germany) and 2\% D(+) Sucrose (AppliChem) in PBS for $30 \mathrm{~min}$ at room temperature and permeabilized in PBST (0.01\% Triton X-100 solution in PBS). Non-specific antibody binding was blocked with $3 \%$ bovine serum albumin (BSA, PanEco, Russia) solution in PBST for an hour at room temperature. Sections were stained with rabbit polyclonal primary antibodies against dystrophin (C357462, 1:200, LS-Bio, USA), utrophin (CAU22354, 1:800, Biomatik, Canada), other DAGC components (ab188873, 1:500, ab189254, 1:1000, Abcam, UK), Flag-epitope (F 7425, 1:200, Sigma, Germany), and Alexa Fluor-labeled secondary antibodies (ab150077, 1:1000, Abcam; A21072, 1:1000, Invitrogen). CD8 + cells were stained with directly-labeled antibodies (42-0081-82, 1:40, ThermoFisher). Nuclei were counterstained with Hoechst 33,342 (ThermoFisher) or DAPI (ThermoFisher). Antibodies and nuclear dyes were diluted in blocking buffer. Incubation with primary antibodies was carried out overnight at 4C, followed by washes in PBST and incubation with secondary antibodies for $1 \mathrm{~h}$. Muscle cryosections were mounted in ProLong Gold antifade medium (Invitrogen, USA). Appropriate negative tissue controls and isotype controls were implemented in the experiments. Fluorescence images were captured on Zeiss LSM 880 and Leica Stellaris 5 confocal microscopes.

In vivo physiological testing in $\mathbf{m d x}$ mice. The wire hanging test was performed for each mouse every week according to the protocol described by Aartsma-Rus and van Putten ${ }^{47}$. A 55-cm-wide 2-mm-thick metallic wire was installed $37 \mathrm{~cm}$ above a layer of bedding. Each mouse was given three trials to hang on the wire by the forelegs with a 30 -s recovery period between trials. The maximum hanging time of the three trials was recorded and used as an outcome measure.

Measurement of isometric muscle force was performed for each mouse at the end of the experiment according to previously described protocols ${ }^{48-50}$. Briefly, the TA muscle was stimulated with $0.2 \mathrm{~ms}$ pulses via two stainless steel electrodes that penetrated the skin on either side of the peroneal nerve near the knee. The muscle was adjusted to an optimum length $(L \mathrm{o})$ to produce the maximum tetanic force $(P \mathrm{O})$. Lo and TA mass were recorded and used for normalization to the physiological cross-sectional area (CSA $=[$ Lo $\times$ density $] /$ mass $)$ and the calculation of the specific tetanic force $(s P o)$.

Susceptibility to eccentric contraction-induced injury was measured during a series of seven eccentric contractions. TA muscle was stimulated $(100 \mathrm{~Hz})$ for $500 \mathrm{~ms}$ at Lo to achieve Po and then stretched at $1 \mathrm{~mm} / \mathrm{s}$ until it was $20 \%$ longer than its $L o$, held at this length for $500 \mathrm{~ms}$, and then returned to its original length at the same rate. The deficit of force at each lengthening contraction $(\mathrm{P} 1-\mathrm{P} 7, \mathrm{P} 1=\mathrm{Po})$ was calculated as $(\mathrm{Po}-\mathrm{P} 2-7) / \mathrm{Po} \times 100 \%$.

Western blotting. $\quad 100 \mathrm{mg}$ of muscle tissue was grinded in liquid nitrogen, resuspended in $1 \mathrm{ml}$ of lysis buffer (110 mM Tris-HCl pH 7,8, $150 \mathrm{mM} \mathrm{NaCl}$, 3\% SDS, 1 mM EDTA, 10\% glycerol, Bromophenol Blue 0.01\% Protease inhibitor cocktail (Roche, Switzerland)) and incubated at $95^{\circ} \mathrm{C}$ for $5 \mathrm{~min} .10-20 \mu \mathrm{g}$ of tissue lysate was loaded per lane of $8 \%$ SDS-polyacrylamide gel. Proteins were wet transferred to a PVDF membrane in Mini Trans-Blot Cell (Bio-Rad, USA). Membrane was blocked in $5 \%$ dry milk in TBS-T overnight. $\mu$ Utrn and $\mu$ Dys were detected using a rabbit polyclonal antibody (CAU22354, 1:500, Biomatic) and mouse monoclonal antibodies (NCL-DYSB, 1:500, Leica, Germany), respectively. Actin and GAPDH were used as loading controls (A2103, 1:10,000, Sigma-Aldrich; ABS16, 1:5000, Sigma-Aldrich). Corresponding species-specific antibodies conjugated with horseradish peroxidase were used as secondary antibodies (1,706,515, 1,706,516, 1:3000, Bio-Rad). Incubation with primary antibodies was carried out for $2 \mathrm{~h}$ at room temperature, followed by washes in TBS-T and incubation with secondary antibodies for $1 \mathrm{~h}$. For humoral immunity assessment, lysates of HEK293T cells transfected with the appropriate transgene were loaded onto a gel. Membrane was blocked in 5\% dry milk in TBS-T for $1 \mathrm{~h}$ followed by overnight incubation with murine serum samples $(1: 100)$ at $4{ }^{\circ} \mathrm{C}$. Protein detection and quantification were performed using the iBright FL1500 Scanner (ThermoFisher) and iBright analysis software.

RT-qPCR analysis. Total RNA was isolated using the ReliaPrep RNA Tissue Miniprep System (Promega, USA) according to the manufacturer's protocol. Purified RNA was treated with RNase-free DNase I (BioLabs, USA) and reverse transcribed using the MMLV RT kit (Evrogen, Russia). Amplification was performed on a Real-Time CFX 96 Touch amplifier (Bio-Rad) using SybrGreen master mix (Evrogen) and primers specific to the transgenes: 5'-TTCAACTACGACGTGTGC-3' and 5'-TCACATGGCCTGGGTCTC-3' for M- $\mu U t r n$, 5'-GAC TAGAAGATTCCTCCAACCA-3' and 5'-TCTGAGTTTCTCCAAATCCAC-3' for $H$ - $\mu U t r n$, 5'-TTCAACTAC GACGTGTGC-3' and 5'-TCACATGGCCTGGGTCTC-3' for Hco- $\mu$ Utrn. Rpl13a, Ap3d1, Csnk2a2 (mouse) and Ankrd27, Hprt1 (rat) were analyzed as housekeeping genes for the normalization of gene expression ${ }^{51,52}$. The following qPCR conditions were used: $94{ }^{\circ} \mathrm{C}-15 \mathrm{~s}, 55 / 61{ }^{\circ} \mathrm{C}-15 \mathrm{~s}, 72{ }^{\circ} \mathrm{C}-15 \mathrm{~s}$ (+ fluorescence measurement). The copy number of utrophin transcripts per $1 \mu \mathrm{g}$ of total cDNA was calculated based on a standard curve built with serial dilutions of the reference plasmid containing the corresponding CDS $(M-\mu U t r n, H-\mu U t r n$, and Hco$\mu(t r n)$. 
Immunofluorescence analysis. Quantitative analysis of immunofluorescence images was performed using the CellProfiler 4.2.1 software in order to compare the degree of DAGC recovery and the numbers of infiltrating CD8 + cells after treatment ${ }^{53}$. The order of selected modules can be found in Supplementary Files S3-S4, and the created pipelines will be available for download at www.cellprofiler.org.

Statistical analysis. Statistical analysis was performed using GraphPad Prism 9 software. Differences between groups were analyzed using the Kruskell-Wallis test, corrected for Dunn's multiple comparisons. Differences between the control $m d x$ group and other groups with $P<0.05$ were considered statistically significant and marked *. Data in the text, tables, and graphs are presented as the mean \pm standard deviation or standard error of the mean (SEM), as stated in the figure legends. The number of measurements is shown in brackets.

Received: 20 September 2021; Accepted: 3 January 2022

Published online: 17 January 2022

\section{References}

1. Mendell, J. R. \& Lloyd-Puryear, M. Report of MDA muscle disease symposium on newborn screening for Duchenne muscular dystrophy. Muscle Nerve 48, 21-26 (2013).

2. Duan, D. Systemic AAV micro-dystrophin gene therapy for duchenne muscular dystrophy. Mol. Ther. 26, 2337-2356 (2018).

3. Mendell, J. R. et al. Dystrophin immunity in Duchenne’s muscular dystrophy. N. Engl. J. Med. 363, 1429-1437 (2010).

4. Gramolini, A. O. \& Jasmin, B. J. Molecular mechanisms and putative signalling events controlling utrophin expression in mammalian skeletal muscle fibres. Neuromuscul. Disord. NMD 8, 351-361 (1998).

5. Clerk, A., Morris, G. E., Dubowitz, V., Davies, K. E. \& Sewry, C. A. Dystrophin-related protein, utrophin, in normal and dystrophic human fetal skeletal muscle. Histochem. J. 25, 554-561 (1993).

6. Tinsley, J. et al. Expression of full-length utrophin prevents muscular dystrophy in mdx mice. Nat. Med. 4, 1441-1444 (1998).

7. Fisher, R. et al. Non-toxic ubiquitous over-expression of utrophin in the mdx mouse. Neuromuscul. Disord. NMD 11, 713-721 (2001).

8. Krag, T. O. B. et al. Heregulin ameliorates the dystrophic phenotype in mdx mice. Proc. Natl. Acad. Sci. U. S. A. 101, 13856-13860 (2004).

9. Moorwood, C. et al. Drug discovery for Duchenne muscular dystrophy via utrophin promoter activation screening. PloS One 6, e26169 (2011).

10. Tinsley, J. M. et al. Daily treatment with SMTC1100, a novel small molecule utrophin upregulator, dramatically reduces the dystrophic symptoms in the mdx mouse. PloS One 6, e19189 (2011).

11. Mattei, E. et al. Utrophin up-regulation by an artificial transcription factor in transgenic mice. PloS One 2, e774 (2007).

12. Pisani, C. et al. Utrophin up-regulation by artificial transcription factors induces muscle rescue and impacts the neuromuscular junction in mdx mice. Biochim. Biophys. Acta Mol. Basis Dis. 1864, 1172-1182 (2018).

13. Amenta, A. R. et al. Biglycan recruits utrophin to the sarcolemma and counters dystrophic pathology in mdx mice. Proc. Natl. Acad. Sci. U. S. A. 108, 762-767 (2011).

14. Ito, M., Ehara, Y., Li, J., Inada, K. \& Ohno, K. Protein-anchoring therapy of biglycan for mdx mouse model of Duchenne muscular dystrophy. Hum. Gene Ther. 28, 428-436 (2017).

15. Cerletti, M. et al. Dystrophic phenotype of canine X-linked muscular dystrophy is mitigated by adenovirus-mediated utrophin gene transfer. Gene Ther. 10, 750-757 (2003).

16. Harper, S. Q. et al. Modular flexibility of dystrophin: implications for gene therapy of Duchenne muscular dystrophy. Nat. Med. 8, 253-261 (2002).

17. Odom, G. L., Gregorevic, P., Allen, J. M., Finn, E. \& Chamberlain, J. S. Microutrophin delivery through rAAV6 increases lifespan and improves muscle function in dystrophic Dystrophin/Utrophin-deficient mice. Mol. Ther. J. Am. Soc. Gene Ther. 16, 1539-1545 (2008).

18. Sonnemann, K. J. et al. Functional substitution by TAT-utrophin in dystrophin-deficient mice. PLoS Med. 6, e1000083 (2009).

19. Kennedy, T. L. et al. Micro-utrophin improves cardiac and skeletal muscle function of severely affected D2/mdx mice. Mol. Ther. Methods Clin. Dev. 11, 92-105 (2018).

20. Song, Y. et al. Non-immunogenic utrophin gene therapy for the treatment of muscular dystrophy animal models. Nat. Med. 25, 1505-1511 (2019).

21. Banks, G. B., Chamberlain, J. S. \& Odom, G. L. Microutrophin expression in dystrophic mice displays myofiber type differences in therapeutic effects. PLoS Genet. 16, e1009179 (2020).

22. Foster, H. et al. Codon and mRNA sequence optimization of microdystrophin transgenes improves expression and physiological outcome in dystrophic mdx mice following AAV2/8 gene transfer. Mol. Ther. J. Am. Soc. Gene Ther. 16, 1825-1832 (2008).

23. Athanasopoulos, T., Foster, H., Foster, K. \& Dickson, G. Codon optimization of the microdystrophin gene for Duchene muscular dystrophy gene therapy. Methods Mol. Biol. Clifton NJ 709, 21-37 (2011).

24. Skopenkova, V. V., Egorova, T. V. \& Bardina, M. V. Muscle-specific promoters for gene therapy. Acta Naturae 13, 47 (2021).

25. Spencer, M. J., Montecino-Rodriguez, E., Dorshkind, K. \& Tidball, J. G. Helper (CD4(+)) and cytotoxic (CD8(+)) T cells promote the pathology of dystrophin-deficient muscle. Clin. Immunol. Orlando Fla 98, 235-243 (2001).

26. Flotte, T. R. et al. Phase 2 clinical trial of a recombinant adeno-associated viral vector expressing a1-antitrypsin: interim results. Hum. Gene Ther. 22, 1239-1247 (2011).

27. Gernoux, G. et al. Muscle-directed delivery of an AAV1 vector leads to capsid-specific T cell exhaustion in nonhuman primates and humans. Mol. Ther. J. Am. Soc. Gene Ther. 28, 747-757 (2020).

28. Ferla, R. et al. Non-clinical safety and efficacy of an AAV2/8 vector administered intravenously for treatment of mucopolysaccharidosis type VI. Mol. Ther. Methods Clin. Dev. 6, 143-158 (2017).

29. Salva, M. Z. et al. Design of tissue-specific regulatory cassettes for high-level rAAV-mediated expression in skeletal and cardiac muscle. Mol. Ther. J. Am. Soc. Gene Ther. 15, 320-329 (2007).

30. Li, X., Eastman, E. M., Schwartz, R. J. \& Draghia-Akli, R. Synthetic muscle promoters: activities exceeding naturally occurring regulatory sequences. Nat. Biotechnol. 17, 241-245 (1999).

31. Hastings, K. E. \& Emerson, C. P. Codon usage in muscle genes and liver genes. J. Mol. Evol. 19, 214-218 (1983).

32. Stedman, H. H., Su, L. T. \& Mitchell, M. A. Microutrophin and uses thereof. (2010).

33. Duan, D. Duchenne muscular dystrophy gene therapy in the canine model. Hum. Gene Ther. Clin. Dev. 26, 57 (2015).

34. Hinderer, C. et al. Severe toxicity in nonhuman primates and piglets following high-dose intravenous administration of an adenoassociated virus vector expressing human SMN. Hum. Gene Ther. 29, 285-298 (2018). 
35. Rodino-Klapac, L. R. et al. Persistent expression of FLAG-tagged micro dystrophin in nonhuman primates following intramuscular and vascular delivery. Mol. Ther. 18, 109-117 (2010).

36. Ronzitti, G., Gross, D.-A. \& Mingozzi, F. Human immune responses to adeno-associated virus (AAV) vectors. Front. Immunol. $11,670(2020)$.

37. Pfizer Presents Initial Clinical Data on Phase $1 \mathrm{~b}$ Gene Therapy Study for Duchenne Muscular Dystrophy (DMD) |Pfizer. https:// www.pfizer.com/news/press-release/press-release-detail/pfizer_presents_initial_clinical_data_on_phase_lb_gene_therapy_study_ for_duchenne_muscular_dystrophy_dmd.

38. Mesnard-Rouiller, L., Bismuth, J., Wakkach, A., Poëa-Guyon, S. \& Berrih-Aknin, S. Thymic myoid cells express high levels of muscle genes. J. Neuroimmunol. 148, 97-105 (2004).

39. Ebihara, S. et al. Differential effects of dystrophin and utrophin gene transfer in immunocompetent muscular dystrophy (mdx) mice. Physiol. Genomics 3, 133-144 (2000).

40. Hakim, C. H. et al. A five-repeat micro-dystrophin gene ameliorated dystrophic phenotype in the severe DBA/2J-mdx model of duchenne muscular dystrophy. Mol. Ther. Methods Clin. Dev. 6, 216-230 (2017).

41. Wang, B. et al. Construction and analysis of compact muscle-specific promoters for AAV vectors. Gene Ther. 15, 1489-1499 (2008).

42. Danilov, K. A. et al. In vitro assay for the efficacy assessment of AAV vectors expressing microdystrophin. Exp. Cell Res. 392, 112033 (2020).

43. Buclez, P.-O. et al. Rapid, scalable, and low-cost purification of recombinant adeno-associated virus produced by baculovirus expression vector system. Mol. Ther. Methods Clin. Dev. 3, 16035 (2016).

44. Zolotukhin, S. et al. Recombinant adeno-associated virus purification using novel methods improves infectious titer and yield. Gene Ther. 6, 973-985 (1999).

45. Aurnhammer, C. et al. Universal real-time PCR for the detection and quantification of adeno-associated virus serotype 2-derived inverted terminal repeat sequences. Hum. Gene Ther. Methods 23, 18-28 (2012).

46. Slaoui, M., Bauchet, A.-L. \& Fiette, L. Tissue sampling and processing for histopathology evaluation. Methods Mol. Biol. Clifton NJ 1641, 101-114 (2017).

47. Aartsma-Rus, A. \& van Putten, M. Assessing functional performance in the mdx mouse model. J. Vis. Exp. JoVE https://doi.org/ $10.3791 / 51303$ (2014).

48. Egorova, T. V. et al. CRISPR/Cas9-generated mouse model of Duchenne muscular dystrophy recapitulating a newly identified large $430 \mathrm{~kb}$ deletion in the human DMD gene. Dis. Model. Mech. 12, 150 (2019).

49. Chan, S., Head, S. I. \& Morley, J. W. Branched fibers in dystrophic mdx muscle are associated with a loss of force following lengthening contractions. Am. J. Physiol. Cell Physiol. 293, C985-992 (2007).

50. Dellorusso, C., Crawford, R. W., Chamberlain, J. S. \& Brooks, S. V. Tibialis anterior muscles in $\mathrm{mdx}$ mice are highly susceptible to contraction-induced injury. J. Muscle Res. Cell Motil. 22, 467-475 (2001).

51. Wang, X. et al. Identification of suitable reference genes for gene expression studies in rat skeletal muscle following sciatic nerve crush injury. Mol. Med. Rep. 19, 4377-4387 (2019).

52. Hildyard, J. C. W., Finch, A. M. \& Wells, D. J. Identification of qPCR reference genes suitable for normalizing gene expression in the mdx mouse model of Duchenne muscular dystrophy. PLOS ONE 14, e0211384 (2019).

53. Jones, T. R. et al. Cell profiler analyst: data exploration and analysis software for complex image-based screens. BMC Bioinf. $\mathbf{9}, 482$ (2008).

\section{Acknowledgements}

We thank the Center for Precision Genome Editing and Genetic Technologies for Biomedicine (Institute of Gene Biology), supported by the Ministry of Science and Higher Education of the Russian Federation (075-15-20191661), for providing the equipment. The research was done using the equipment of the Core Centrum of the Institute of Developmental Biology RAS. The work was supported by The Foundation for Assistance to Small Innovative Enterprises in Science and Technology (grant number GRNTIS5/42610).

\section{Author contributions}

S.A.V. S.V.V.- investigation, formal analysis, data curation, visualization, writing original draft; P.A.V.- investigation, formal analysis, R.D.A.-conceptualization, Investigation, V.S.G., V.O.A., S.A.A., S.I.M., S.V.O.-investigation, E.T.V.-conceptualization, project administration, funding acquisition, writing- review and editing; B.M.V.-supervision, validation, writing- review and editing. All authors reviewed the manuscript.

\section{Competing interests}

The authors declare no competing interests.

\section{Additional information}

Supplementary Information The online version contains supplementary material available at https://doi.org/ 10.1038/s41598-022-04892-x.

Correspondence and requests for materials should be addressed to T.V.E.

Reprints and permissions information is available at www.nature.com/reprints.

Publisher's note Springer Nature remains neutral with regard to jurisdictional claims in published maps and institutional affiliations.

(c) (i) Open Access This article is licensed under a Creative Commons Attribution 4.0 International License, which permits use, sharing, adaptation, distribution and reproduction in any medium or format, as long as you give appropriate credit to the original author(s) and the source, provide a link to the Creative Commons licence, and indicate if changes were made. The images or other third party material in this article are included in the article's Creative Commons licence, unless indicated otherwise in a credit line to the material. If material is not included in the article's Creative Commons licence and your intended use is not permitted by statutory regulation or exceeds the permitted use, you will need to obtain permission directly from the copyright holder. To view a copy of this licence, visit http://creativecommons.org/licenses/by/4.0/.

(c) The Author(s) 2022 